David J. Unger*

\title{
The yield condition strongly influences the formation of Dugdale plastic strips ahead of crack tips under tensile plane stress loading conditions
}

\begin{abstract}
A finite element analysis indicates a good correlation between the Dugdale plastic strip model and a linear elastic/perfectly plastic material under plane stress loading conditions for a flow theory of plasticity based on the Tresca yield condition. A similar analysis under the von Mises yield condition reveals no plastic strip formation.
\end{abstract}

Keywords: computational; Dugdale model; Tresca yield.

*Corresponding author: David J. Unger, Department of Mechanical and Civil Engineering, University of Evansville, 1800 Lincoln Avenue, Evansville, IN 47722, USA, e-mail: du2@evansville.edu

\section{Background}

The Dugdale plastic strip model [1] has become a standard representation of the plastic zone under plane stress loading conditions for mode I cracks in materials exhibiting elastic/perfectly plastic behavior. This model is based on a linear elastic analysis where the plastic zone is introduced as a boundary condition on traction along the crack faces near its tip. The investigators in [2-4] provided a rational basis for this model based on the slip line theory and indicated an alternative plastic zone response called the hinge model for plane strain loading conditions. They also conducted some of the early experiments illustrating both types of elastic-plastic behavior in the plane. A good correlation [4] was noted between the experiment $[4,5]$ and the Dugdale model under plane stress loading conditions for materials where strain hardening was minimal.

Although many elastic-plastic finite element analyses have been conducted under the von Mises yield condition for crack or notch problems, only two have been conducted under the Tresca yield condition. The earliest [6] was for plane strain loading conditions, whereas the latter [7] was for plane stress loading conditions. Both analyses were for materials exhibiting a linear elastic/perfectly plastic response. The plane strain finite element analysis exhibited no plastic strip formation, whereas the plane stress finite element analysis indicated the beginning of one. Here, results are reported for the first time for load levels higher than those used in [7], that is, when plastic strip development is extensive. Small strain assumptions were used in both analyses, and the load was incrementally applied to its maximum value.

The Dugdale plastic zone length $c$ (see Figure 1) is given in [8] by

$$
\frac{c}{a}=\sec \left(\frac{\pi \sigma_{\infty}}{2 \sigma_{0}}\right),
$$

where $a$ is the crack length, $\sigma_{\infty}$ is a remotely applied tensile traction, and $\sigma_{0}$ is the yield strength of an elastic/ perfectly plastic material.

The details of the finite element modeling are provided in [7] and will not be repeated here. Note a typographical error (missing $y$ ) exists in the right-hand side of Eq. (5) of [7].

The only difference in the material properties used here from those used in [7] is that the yield strength is onetenth of the previous value. The remotely applied tensile traction was proportionally adjusted in Figure 1A, so that a correlation can be made between the two analyses for a similar ratio of $\sigma_{\infty} / \sigma_{0}$. A comparison of Figure $1 \mathrm{~A}$ and Figure 3 of [7] indicates virtually no change in the size or shape of the plastic zone for the same ratio of applied load to yield strength. The elastic-plastic boundary can be taken in these figures as the farthest extent of the equivalent plastic strain contours, which are represented by various shades of gray. The square grid imposed on the figures indicates the relative size of the quad 4 element that was used in the NASTRAN 70.5 code with an MSC PATRAN graphical interface (MSC Software Corporation, Santa Ana, CA, USA).

\section{Results}

In Figure 2, the normalized plastic zone length $c / a$ is plotted versus the normalized load $\sigma_{\infty} / \sigma_{0}$ for three different values of the normalized load that were analyzed in the finite element program. It is noted that the plastic strip 
A $y$

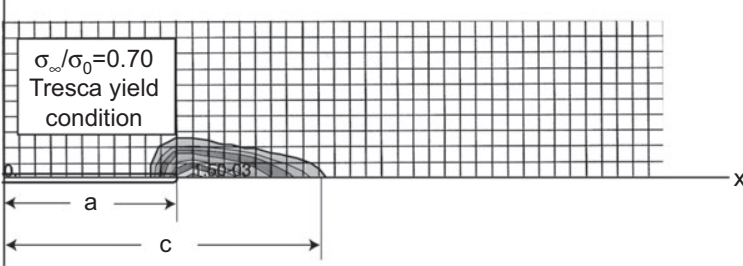

B

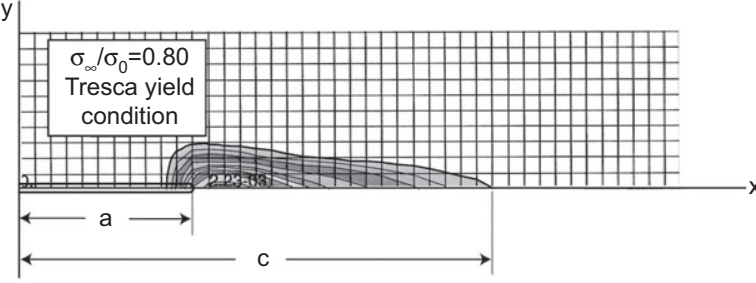

C

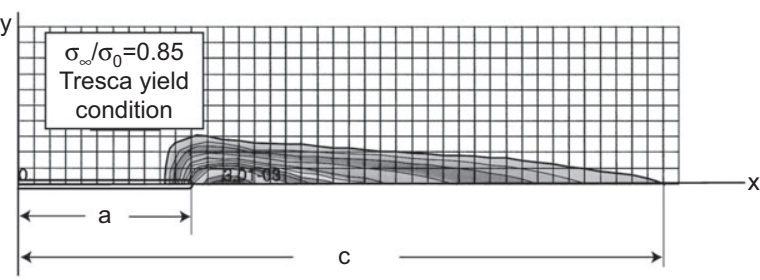

D

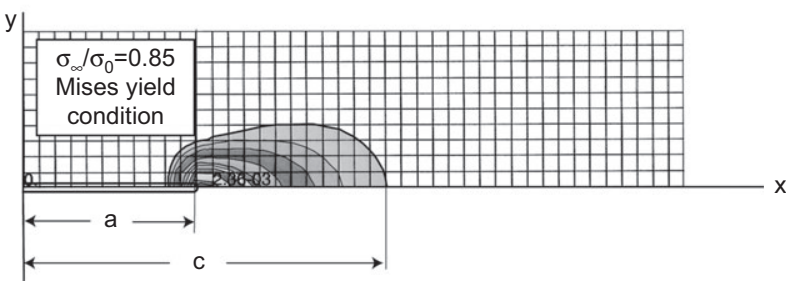

Figure 1 Effect of yield condition and applied load on equivalent plastic strain contours.

regions become apparent in Figure $1 \mathrm{~A}-\mathrm{C}$ as higher levels of load are attained. For comparison, a continuous plot of the analytical Dugdale relationship (1) is also shown. The finite element predictions fall below the locus of the Dugdale model but have a similar trend. These differences may be attributed to the fact the finite element program uses a flow theory of plasticity outside the elastic region, whereas the Dugdale model is based entirely on linear elasticity. In the Dugdale model, the plastic response is represented by a boundary condition on traction that satisfies the Tresca yield condition along a portion of the mathematical crack of length $c$. The physical crack length $a$ in the Dugdale model is represented by the traction-free portion of the mathematical crack. In the finite element program, the physical crack is of length $a$, whereas $c$ represents the extent of the plastic zone as measured from the origin. In the Dugdale model, there is discontinuity in displacement between the two surfaces in the cohesive zone region, $a \leq x \leq c$ which is physically unrealistic. In the finite element model, there is no such discontinuity

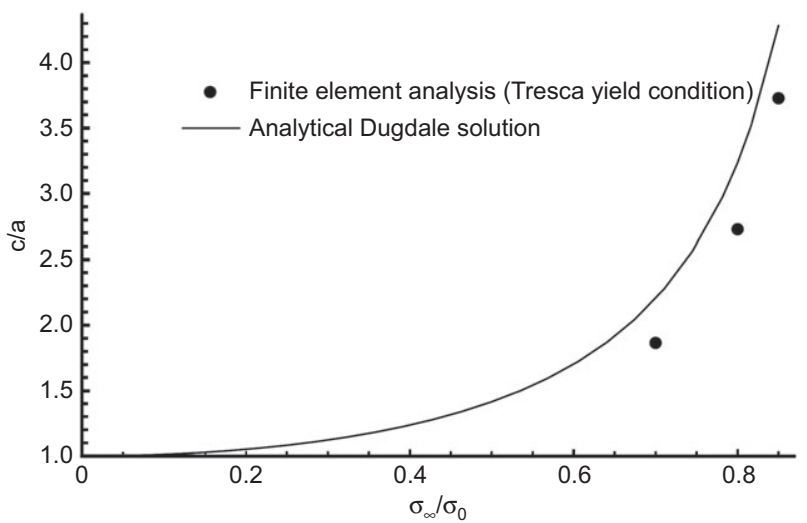

Figure 2 Comparison of normalized plastic zone length to normalized load between numerical and analytical solutions.

in displacement in the plastic region along the crack axis. Lastly, the analytical Dugdale model is for an infinite plate, whereas numerical methods must approximate the far-field boundary with sufficiently large but finite dimensions in comparison to the crack length and plastic zone.

To demonstrate that the von Mises yield condition does not produce plastic strip regions under plane stress loading conditions, a similar finite element analysis was performed for the highest load level that was previously used for the Tresca yield condition. The results of the two different analyses are illustrated in Figure 1C and D. The shape of the plastic region is not elongated for the von Mises analysis as for the finite element analysis using the Tresca yield condition. It is qualitatively similar to that obtained in [9] for a linear elastic/perfectly plastic finite element analysis under the von Mises yield condition and the associated Prandtl-Reuss plastic flow equations. The coarseness of the finite element mesh used here in comparison to the fine mesh used in the custom finite element program developed in [9] may account for those differences that do exist, such as no plasticity behind the crack tip [9].

The behavior of the analyses reported here clearly indicates the tendency for plastic strip zones to form under the Tresca yield condition but not under the von Mises yield condition, assuming plane stress loading conditions and linear elastic/perfectly plastic material.

Acknowledgements: This research was performed while the author was a visiting associate professor in the Department of Mechanical Engineering and Engineering Mechanics at the Michigan Technological University, Houghton, Michigan. The author is grateful to both the Department and the College of Engineering for the opportunity to perform this analysis and for purchasing a license for MSC PATRAN/NASTRAN for this purpose. 


\section{References}

[1] Dugdale DS. J. Mech. Phys. Solids 1960, 8, 100-104.

[2] Hahn GT, Rosenfield RA. Acta Metal. 1965, 13, 293-306.

[3] Rosenfield RA, Dai PK, Hahn GT. Proceedings of the First International Conference on Fracture, Vol. 1. Yokobori T, Kawasaki T, Swedlow JL, Eds. Sendai, Japan: Japanese Society for Strength and Fracture Mechanics, 1966, pp. 223-258.

[4] Hahn GT, Rosenfield RA. Applications Related Phenomena in Titanium Alloys, STP 432. Philadelphia, PA: American Society for Testing and Materials, 1968, pp. 5-32.
[5] Gerberich WW. Exp. Mech. 1964, 4, 335-344.

[6] Owen DRJ, Nayak GC, Kfouri AP. Int. J. Numer. Methods Eng. 1973, 6, 63-73.

[7] Unger DJ. Mech. Res. Commun. 2007, 34, 325-330.

[8] Burdekin FM, Stone DEW. J. Strain Anal. 1966, 1, 145-153.

[9] Narasimhan R, Rosakis AJ. J. Mech. Phys. Solids 1988, 36, 77-117. 